\title{
ON THE EXISTENCE OF AN INERTIAL MANIFOLD FOR A DECONVOLUTION MODEL OF THE 2D MEAN BOUSSINESQ EQUATIONS
}

\author{
LUCA BISCONTI, DAVIDE CATANIA
}

\author{
LuCA Bisconti \\ Università degli Studi di Firenze \\ Dipartimento di Matematica e Informatica "U. Dini" \\ Via S. Marta 3, I-50139 Firenze, Italia \\ Davide CATANia \\ Università degli Studi di Brescia \\ Sezione Matematica (DICATAM) \\ Via Valotti 9, I-25133 Brescia, Italia
}

\begin{abstract}
We show the existence of an inertial manifold (i.e. a globally invariant, exponentially attracting, finite-dimensional manifold) for the approximate deconvolution model of the 2D mean Boussinesq equations. This model is obtained by means of the Van Cittern approximate deconvolution operators, which is applied to the 2D filtered Boussinesq equations.
\end{abstract}

\section{Introduction}

In what follows we prove the existence of an inertial manifold for a regularized version of the two-dimensional viscous Boussinesq system

$$
\begin{aligned}
& \partial_{t} \boldsymbol{u}-\nu \Delta \boldsymbol{u}+\nabla \cdot(\boldsymbol{u} \otimes \boldsymbol{u})+\nabla \pi=\theta \boldsymbol{e}_{2} \\
& \partial_{t} \theta-\kappa \Delta \theta+\boldsymbol{u} \cdot \nabla \theta=f \\
& \nabla \cdot \boldsymbol{u}=0 \\
& \left.(\boldsymbol{u}, \theta)\right|_{t=0}=\left(\boldsymbol{u}_{0}, \theta_{0}\right)
\end{aligned}
$$

where $\nu>0$ and $\kappa>0$ are viscosities, $\boldsymbol{u}=\left(u_{1}, u_{2}\right)$ is the velocity field, $\theta$ may be interpreted physically as a density, $\pi$ is the cinematic pressure, and $\boldsymbol{e}_{2}:=(0,1)^{T}$ where $\left\{\boldsymbol{e}_{1}, \boldsymbol{e}_{2}\right\}$ is the canonical basis of $\mathbb{R}^{2}$. Here, we also consider an external action $f=f(\boldsymbol{x})$, independent of time, forcing the evolution of $\theta$.

The Boussinesq equations are employed, for instance, as a mathematical scheme to describe Newtonian fluids whenever density, salinity concentration, or temperature variations (according to the meaning of $\theta$ ) play significant roles (see, e.g., 4, 5, 9] for some recent papers on this subject).

In performing our analysis we follow closely what has been done in [1] where the existence of inertial manifolds has been proved in the case of two regularizing models for the 2D NavierStokes equations (NSEs). Differently from reference [1, here we consider a turbulence model that converges to 2D mean Navier-Stokes-like equations, namely the 2D mean Boussinesq system, when

2000 Mathematics Subject Classification. 35Q35, 35Q30, 37L30, 76D03, 76F20, 76F65.

Key words and phrases. Boussinesq equations, Navier-Stokes equations, turbulent flows, Large Eddy Simulation (LES), deconvolution models, inertial manifold, global attractor. 
the deconvolution parameter $N$ goes to infinity (see (5), below). Further, we take into account a double filtered system, that is a model in which both equations for $\boldsymbol{u}$ and $\theta$ are somehow regularised by an application of the inverse of the Helmholtz operator (see (6)-(9) below).

We set $A=\mathrm{I}-\alpha^{2} \Delta, \overline{\boldsymbol{u}}=A^{-1} \boldsymbol{u}$, and take $g(\boldsymbol{x})=\bar{f}(\boldsymbol{x})=A^{-1} f(\boldsymbol{x})$. Filtering the equations (11) -(41), we obtain what we call the "mean Boussinesq equations", i.e.

$$
\begin{aligned}
& \partial_{t} \overline{\boldsymbol{u}}-\nu \Delta \overline{\boldsymbol{u}}+\nabla \cdot \overline{(\boldsymbol{u} \otimes \boldsymbol{u})}+\nabla \bar{\pi}=\bar{\theta} \boldsymbol{e}_{2} \\
& \partial_{t} \bar{\theta}-\kappa \Delta \bar{\theta}+\nabla \cdot \overline{(\theta \boldsymbol{u})}=\bar{f} \\
& \nabla \cdot \overline{\boldsymbol{u}}=0 \\
& \left.(\overline{\boldsymbol{u}}, \bar{\theta})\right|_{t=0}=\left(\overline{\boldsymbol{u}_{0}}, \overline{\theta_{0}}\right)
\end{aligned}
$$

where $\boldsymbol{u} \otimes \boldsymbol{u}:=\left(u_{1} \boldsymbol{u}, u_{2} \boldsymbol{u}\right)$, and we supply this problem, which is equivalent to (1)-(4), with periodic boundary conditions (i.e., the torus $\Omega=\mathbb{T}^{2}$ is the considered domain).

Here, we consider the Approximate Deconvolution Model (ADM), introduced by Adams and Stolz [2, 23, 24] (see also [21, 8, 6, 9]); by following this scheme we approximate the filtered bi-linear terms as follows:

$$
\overline{(\boldsymbol{v} \otimes \boldsymbol{v})} \sim \overline{\left(D_{N}(\overline{\boldsymbol{v}}) \otimes D_{N}(\overline{\boldsymbol{v}})\right)} \text { and } \overline{(\varphi \boldsymbol{v})} \sim \overline{\left(D_{N}(\bar{\varphi}) D_{N}(\overline{\boldsymbol{v}})\right)},
$$

where $\boldsymbol{v}$ and $\varphi$ play the role of the variables $\boldsymbol{u}$ and $\theta$, respectively, and the filtering operator $G_{\alpha}$ is defined by the Helmholtz filter (see, e.g., [7, 8, 21, 3], see also [10, 11] for an analogous case involving an anisotropic horizontal filter), with $\overline{(\cdot)}=G_{\alpha}(\cdot)$ and $G_{\alpha}:=\left(I-\alpha^{2} \Delta\right)^{-1}$. Here, $D_{N}$ is the deconvolution operator, which is constructed using the Van Cittert algorithm (see, e.g, [21]) and is formally defined by

$$
D_{N}:=\sum_{n=0}^{N}\left(I-G_{\alpha}\right)^{n} \text { with } N \in \mathbb{N} .
$$

The ADM we analyze here is defined by

$$
B(\boldsymbol{w}, \boldsymbol{w}):=\overline{D_{N}(\boldsymbol{w}) \otimes D_{N}(\boldsymbol{w})}, \quad \mathcal{B}(\rho, \boldsymbol{w}):=\overline{D_{N}(\rho) D_{N}(\boldsymbol{w})},
$$

and the considered system (see [9, 8] for the global existence and well-posedness of the 3D case) is the following

$$
\begin{aligned}
& \partial_{t} \boldsymbol{w}-\nu \Delta \boldsymbol{w}+\nabla \cdot \overline{D_{N} \boldsymbol{w} \otimes D_{N} \boldsymbol{w}}+\nabla q=\rho \boldsymbol{e}_{2}, \\
& \partial_{t} \rho-k \Delta \rho+\nabla \cdot \overline{D_{N} \rho D_{N} \boldsymbol{w}}=g, \\
& \nabla \cdot \boldsymbol{w}=0 \\
& \boldsymbol{w}(0, x)=\overline{\boldsymbol{u}_{0}}(x), \text { and } \rho(0, t)=\overline{\theta_{0}}(x) .
\end{aligned}
$$

This system of partial differential equations (PDEs) has a dissipative nature and so, energy associated with weak solutions decreases during time. Consequently, we can presume the existence of a global attractor $\mathcal{A}$, i.e. a compact and non-empty subset of the phase space $\mathcal{H}$ (with metric $d$ ), of the initial data, which is invariant with respect to the action of the semigroup $S(t)$ associated with the system itself (see, e.g., [25, 12, 16, 26]). Again, $\mathcal{A}$ has the property of attracting images, under the action of $S(t)$, of any bounded subsets $B \subseteq \mathcal{H}$ when time $t$ goes to $+\infty$ (i.e., $\left.\lim _{t \rightarrow+\infty} \mathrm{d}(S(t) B, \mathcal{A})=0\right)$. Consequently, $\mathcal{A}$ consists of the full trajectories of the considered system and contains all the related "non-trivial" dynamics (see, e.g., [26]).

In fact, in the case of system (6) -(9), we can say more because the associated dynamics is effectively finite dimensional and it can be completely analyzed by using a suitable system of 
ordinary differential equations (ODEs). We prove this fact in the main result of the paper, i.e. Theorem 4.4 by considering suitably modified equations (see later on).

By definition (see [17, 19]), an inertial manifold for an evolution equation is a finite-dimensional smooth submanifold $\mathcal{M}$ of the phase space $\mathcal{H}$, which is invariant with respect to the action of the semigroup $S(t)$, it contains the global attractor $\mathcal{A}$, and it is also such that any trajectory starting outside of $\mathcal{M}$ approaches, exponentially fast, some other trajectory belonging to $\mathcal{M}$. If this object exists, by restricting the evolution equation (here, in fact, we think of a single or a system of PDEs) to $\mathcal{M}$, we get a finite system of ODEs that captures the dynamics on the attractor. This system of ODEs is called the "inertial form of the given evolutionary system". As a result, the dynamical properties of the solution of the evolutionary PDE (or of the system of PDEs), which is an infinite-dimensional system, can be analyzed by treating a finite-dimensional scheme, which is the corresponding inertial form.

As remarked in [1], the original motivations for the development of the theory of inertial manifolds were mainly related to the analysis of the NSEs. Nevertheless, as far as we know, the problem about the existence of inertial manifolds for the 2D NSEs is still open. As a partial answer in this direction, the existence of inertial manifolds has been proved for some " $\alpha$-models"; to be precise, for the $2 \mathrm{D}$ Bardina model and for the 2D modified Leray- $\alpha$ (see [1]).

The paper is organized as follows: in Section 2 we introduce preliminary notions and the functional setting. In Section 3 we provide some a-priori estimates that highlight the dissipative nature of the considered equations. Section 4 is devoted to prove the existence of absorbing sets in suitable Hilbert spaces, as well as to show the existence of an inertial manifold for a modification of the approximate deconvolution system (6)-(9).

\section{BASIC FACTS AND NOTATION}

We introduce the functional setting, and we recall the definition and the main properties of the deconvolution operator $D_{N}$.

Denote by $x:=\left(x_{1}, x_{2}\right) \in \mathbb{R}^{2}$ a generic point in $\mathbb{R}^{2}$. Given $L \in \mathbb{R}_{+}^{\star}:=\{x \in \mathbb{R}: x>0\}$, consider ] $0, L\left[^{2} \subset \mathbb{R}^{2}\right.$ and $\mathcal{T}_{2}:=2 \pi \mathbb{Z}^{2} / L ; \Omega=\mathbb{T}^{2}$ is the torus defined by $\mathbb{T}^{2}:=\left(\mathbb{R}^{2} / \mathcal{T}_{2}\right)$. For $p, k \in \mathbb{N}$, we consider the standard Lebesgue spaces $L^{p}=L_{p e r}^{p}=L^{p}\left(\mathbb{T}^{2}\right)$ and Sobolev spaces $W^{k, p}=W^{k, p}\left(\mathbb{T}^{2}\right)$, with $H^{k}:=W^{k, 2}$, in the periodic setting. We denote by $\|\cdot\|$ the $L^{2}\left(\mathbb{T}^{2}\right)$-norm and the associated operator norms, and we always impose the zero mean condition on the considered fields. In the sequel, we will use the same notation for scalar and vector-valued functions, since no ambiguity occurs. Also, dealing with divergence-free vector fields, we also define, for a generic exponent $s \geq 0$, the following spaces

$$
H_{s}:=\left\{\boldsymbol{v}: \mathbb{T}^{2} \rightarrow \mathbb{R}^{2}: \boldsymbol{v} \in\left(H^{s}\right)^{2}, \nabla \cdot \boldsymbol{v}=0, \int_{\mathbb{T}^{2}} \boldsymbol{v} d x=0\right\} .
$$

When $0 \leq s \leq 1$, the condition $\nabla \cdot \boldsymbol{v}=0$ must be understood in weak sense. Let $X$ be a real Banach space with norm $\|\cdot\|_{X}$. We will use the customary Bochner spaces $L^{q}(0, T ; X)$, with norm denoted by $\|\cdot\|_{L^{q}(0, T ; X)}$. We denote by $P_{\sigma}: L_{\text {per }}^{2} \rightarrow H:=H^{0}$ the Helmholtz-Leray orthogonal projection operator, and by $\mathbf{A}=-P_{\sigma} \Delta$ the Stokes operator. Since we are dealing with periodic-boundary conditions, we have that

$$
\mathbf{A} u=-P_{\sigma} \Delta u=-\Delta u, \text { for all } u \in D(\mathbf{A}) .
$$

For $v \in H^{s}$, we can expand the fields as $v(x)=\sum_{k \in \mathcal{T}_{2}{ }^{\star}} \widehat{v}_{k} e^{i k \cdot x}$, where $k \in \mathcal{T}_{2}^{\star}$, and the Fourier coefficients are defined by $\widehat{v}_{k}=1 /\left|\mathbb{T}^{2}\right| \int_{\mathbb{T}^{2}} v(x) e^{-i k \cdot x} d x$. The magnitude of $k$ is given by $|k|^{2}:=$ 
$\left(k_{1}\right)^{2}+\left(k_{2}\right)^{2}$. The $H^{s}$ norms are defined by $\|v\|_{s}^{2}:=\sum_{k \in \mathcal{T}_{2}^{\star}}|k|^{2 s}\left|\widehat{v}_{k}\right|^{2}$, where $\|v\|_{0}^{2}:=\|v\|^{2}$. The inner products associated to these norms are $(w, v)_{H^{s}}:=\sum_{k \in \mathcal{T}_{2}^{*}}|k|^{2 s} \widehat{w}_{k} \cdot \overline{\mathrm{v}}_{k}$, where $\overline{\widehat{v}_{k}}$ denotes the complex conjugate of $\widehat{v}_{k}$. To have real valued vector fields, we impose $\widehat{v}_{-k}=\overline{\widehat{v}_{k}}$ for any $k \in \mathcal{T}_{2}^{\star}$ and for any field denoted by $v$. When $s$ is an integer, $\|v\|_{s}^{2}=\left\|\nabla^{s} v\right\|^{2}$ and also, for general $s \in \mathbb{R}$, $\left(H^{s}\right)^{\prime}=H^{-s}$, (see e.g. [14]). All these considerations can be adapted straightforwardly to the case of the spaces $H_{s}$; we finally characterize $H_{s} \subseteq\left(W^{s, 2}\left(\mathbb{T}^{2}\right)\right)^{2}$ as follows:

$$
H_{s}=\left\{\boldsymbol{v}=\sum_{|k| \geq 1} \widehat{\boldsymbol{v}}_{k} e^{i k \cdot x}: \sum_{|k| \geq 1}|k|^{2 s}\left|\widehat{\boldsymbol{v}}_{k}\right|^{2}<\infty, k \cdot \widehat{\boldsymbol{v}}_{k}=0, \widehat{\boldsymbol{v}}_{-k}=\overline{\widehat{\boldsymbol{v}}_{k}}\right\} .
$$

In particular, we denote $\left(H_{s}\right)^{\prime}$ by $H_{-s}$.

We will denote by $C$ generic constants, which may change from line to line, but which are independent of the diffusion coefficient $\epsilon$, the deconvolution parameter $N$ and of the solution of the equations we are considering.

Let us now briefly recall the properties of the Helmholtz filter. Let $\alpha>0$ be a given fixed number and, for $\boldsymbol{\omega} \in H_{s}, s \geq-1$, let us denote by $(\overline{\boldsymbol{\omega}}, \pi) \in H_{s+2} \times H^{s+1}$, the unique solution of the following Stokes-like problem:

$$
\begin{aligned}
& \overline{\boldsymbol{\omega}}-\alpha^{2} \Delta \overline{\boldsymbol{\omega}}+\nabla \pi=\boldsymbol{\omega} \text { in } \mathbb{T}^{2}, \\
& \nabla \cdot \overline{\boldsymbol{\omega}}=0 \text { in } \mathbb{T}^{2},
\end{aligned}
$$

with $\int_{\mathbb{T}^{2}} \overline{\boldsymbol{\omega}} d x=0$ and $\int_{\mathbb{T}^{2}} \pi d x=0$. The velocity component of $(\overline{\boldsymbol{\omega}}, \pi)$ is denoted also by $\overline{\boldsymbol{\omega}}=G_{\alpha}(\boldsymbol{\omega})$ and $A_{1}:=G_{\alpha}^{-1}$. Consider an element $\boldsymbol{\omega} \in H_{s}$ and take its expansion in terms of Fourier series as $\boldsymbol{\omega}=\sum_{k \in \mathcal{T}_{2}^{\star}} \widehat{\boldsymbol{\omega}}_{k} e^{i k \cdot x}$, so that inserting this expression in (11) and looking for $(\bar{\omega}, \pi)$, we get

$$
\overline{\boldsymbol{\omega}}=\sum_{k \in \mathcal{T}_{2}^{\star}} \frac{1}{1+\alpha^{2}|k|^{2}} \widehat{\boldsymbol{w}}_{k} e^{i k \cdot x}=G_{\alpha}(\boldsymbol{\omega}), \text { and } \pi=0 .
$$

For a scalar function $\zeta$ we denote by $\bar{\zeta}$ the solution of the pure Helmholtz problem

$$
-\alpha^{2} \Delta \bar{\zeta}+\bar{\zeta}=\zeta \text { in } \mathbb{T}^{2}
$$

where $A_{2} \bar{\zeta}:=-\alpha^{2} \Delta \bar{\zeta}+\bar{\zeta}$. Further, taking $\zeta \in H^{s}$ the expression of $\bar{\zeta}$ in terms of Fourier series can be retrieved, formally, by (13) substituting $\zeta$ in place of $w$.

In what follows, in order to keep the notation compact, we use the same symbol $A$ for the operators $A_{1}$ and $A_{2}$, distinguishing the two situations only when it is required by the context. According to the above facts, the deconvolution operator $D_{N}$ is actually given by $D_{N}=\sum_{n=0}^{N}(I-$ $\left.A^{-1}\right)^{n}, N \in \mathbb{N}$, with $A$ defined by (11), when it is acting on the elements of $H_{s}$ and, by relation (13), in the case of the scalar functions in $H^{s}$.

Let us recall that, in the filtered equations (6)-(7), the symbol " -" (i.e " $\div$ ") denotes the pure Helmholtz filter, applied component-wise to the various vector and tensor fields. Referring to the right-hand side of equation (6), since $\boldsymbol{e}_{2}$ is a constant vector, then we have that $G_{\alpha}\left(\theta \boldsymbol{e}_{2}\right)=\overline{\theta \boldsymbol{e}_{2}}=$ $\bar{\theta} \boldsymbol{e}_{2}=G_{\alpha}(\theta) \boldsymbol{e}_{2}$ and $A\left(\overline{\theta \boldsymbol{e}_{2}}\right)=A(\bar{\theta}) \boldsymbol{e}_{2}$ (where the meaning of $A$ is understood in the sense stated above). Also, for brevity, in the sequel we omit the explicit dependence of $G_{\alpha}$ on $\alpha$ (we write $G$ in place of $G_{\alpha}$ ) and we will always use the notation $G=A^{-1}=\left(I-\alpha^{2} \Delta\right)^{-1}$.

The deconvolution operator $D_{N}$ is constructed thanks to the Van Cittert algorithm; the reader can find an exhaustive description and analysis of the Van Cittert algorithm and its variants in [21]. Here, we only report the properties needed to describe the considered model. Let $\omega \in H_{s}$ (or 
$\omega \in H^{s}$ ), starting from the expression (12), we can write the deconvolution operator in terms of Fourier series as follows

$$
\begin{gathered}
\widehat{D}_{N}(k)=\sum_{n=0}^{N}\left(\frac{\alpha^{2}|k|^{2}}{1+\alpha^{2}|k|^{2}}\right)^{n}=\left(1+\alpha^{2}|k|^{2}\right) \varrho_{N, k} \\
\varrho_{N, k}=1-\left(\frac{\alpha^{2}|k|^{2}}{1+\alpha^{2}|k|^{2}}\right)^{N+1} \text { and } D_{N}(\omega)=\sum_{k \in \mathcal{T}_{2}^{\star}} \widehat{D}_{N}(k) \widehat{\omega}_{k} e^{i k \cdot x} .
\end{gathered}
$$

The properties verified by $\widehat{D}_{N}$ are summarized in [8, Lemma 2.1] and [8, Lemma 2.2] and, even if we do not use them directly, we recall here the main points for the reader's convenience:

Lemma 2.1. For each fixed $k \in \mathcal{T}_{2}$,

$$
\widehat{D}_{N}(k) \rightarrow 1+\alpha^{2}|k|^{2}=\widehat{A}_{k} \text {, as } N \rightarrow+\infty \text {, even if not uniformly in } k \text {. }
$$

Further, for each $N \in \mathbb{N}$ the operator $D_{N}: H_{s} \rightarrow H_{s}$ is self-adjoint, it commutes with differentiation, and the following properties hold true:

$$
\begin{aligned}
& 1 \leq \widehat{D}_{N}(k) \leq N+1, \forall k \in \mathcal{T}_{2}^{\star}, \\
& \widehat{D}_{N}(k) \cong(N+1) \frac{1+\alpha^{2}|k|^{2}}{\alpha^{2}|k|^{2}} \text { for large }|k|, \\
& \widehat{D}_{N}(k) \leq 1+\alpha^{2}|k|^{2}=\widehat{A}_{k}, \forall k \in \mathcal{T}_{2}^{\star}, \alpha>0, \\
& \text { the map } \omega \mapsto D_{N}(\omega) \text { is an isomorphism s.t. }\left\|D_{N}\right\|_{H_{s}}=O(N+1), \forall s \geq 0, \\
& \lim _{N \rightarrow+\infty} D_{N}(\omega)=A \omega \text { in } H_{s} \forall s \in \mathbb{R} \text { and } \omega \in H_{s+2} .
\end{aligned}
$$

This lemma can be readily extended to the case of spaces $H^{s}$.

\section{Preliminary estimates}

In what follows we provide a priori estimates needed to study the long-time dynamics of the solution of system (6)-(9). In particular, we are required to justify the existence of absorbing balls for the dynamical system induced by equation (6) -(9), in various spaces of functions. The controls given in the sequel are formal, but it is possible to prove them rigorously, by using and adapted Galerkin approximation procedure that now we briefly illustrate.

3.1. Galerkin scheme. We give some details about the construction of the approximate solutions, which is however classical (for more details see, e.g., [15, 22, 8, 9]). Let be given $m \in \mathbb{N} \backslash\{0\}$ and define

$$
\begin{aligned}
& V^{m}=\left\{\vartheta \in H^{1}: \int_{\mathbb{T}^{3}} \vartheta(x) e^{-i k \cdot x} d x=0, \forall k \text { with }|k|>m\right\}, \\
& \mathbf{V}_{m}=\left\{\mathbf{w} \in H_{1}: \int_{\mathbb{T}^{3}} \mathbf{w}(x) e^{-i k \cdot x} d x=0, \forall k \text { with }|k|>m\right\},
\end{aligned}
$$

and let $\left\{E_{j}\right\}_{j=1, \ldots, d_{m}}$ and $\left\{\mathbf{E}_{j}\right\}_{j=1, \ldots, \delta_{m}}$ be orthogonal bases of $V^{m}$ and $\mathbf{V}_{m}$ respectively. Without loss of generality, we can assume that the $E_{j}$ 's are eigen-functions of the operator $I-\alpha^{2} \Delta$ introduced in (13) as well as the $\mathbf{E}_{j}$ 's are eigen-functions of the Stokes-like operator associated to (11). Further, the $E_{j}$ 's and $\mathbf{E}_{j}$ 's are supposed to have unitary norm. We denote by $P_{m}$ the orthogonal projection 
from $H^{1}$ onto $V^{m}$ and, similarly, by $\mathbf{P}_{m}$ the the orthogonal projection from $H_{1}$ onto $\mathbf{V}_{m}$. For every positive integer $m$, we look for an approximate solution of equations (6)-(9) of the form

$$
\rho_{m}(t, x)=\sum_{j=1}^{d_{m}} \rho_{m, j}(t) E_{j}(x) \text { and } \boldsymbol{w}_{m}(t, x)=\sum_{j=1}^{\delta_{m}} \boldsymbol{w}_{m, j}(t) \mathbf{E}_{j}(x) .
$$

Thanks to the Cauchy-Lipschitz Theorem, we can prove the existence of a unique $C^{1}$ maximal solution $\left(\boldsymbol{w}_{m}(t), \rho_{m}(t)\right) \in \mathbf{V}_{m} \times V^{m}$ for all $t \in\left[0, T_{m}\right)$ where $T_{m}>0$ is the maximal existence time, to the system

$$
\begin{aligned}
& \int_{\mathbb{T}^{2}} \partial_{t} \boldsymbol{w}_{m} \cdot \boldsymbol{v}-\int_{\mathbb{T}^{2}} G\left(D_{N}\left(\boldsymbol{w}_{m}\right) \otimes D_{N}\left(\boldsymbol{w}_{m}\right)\right): \nabla \boldsymbol{v} \\
& \quad+\nu \int_{\mathbb{T}^{2}} \nabla \boldsymbol{w}_{m}: \nabla \boldsymbol{v}=\int_{\mathbb{T}^{2}} \rho_{m} \mathbf{e}_{2} \cdot \boldsymbol{v}, \\
& \int_{\mathbb{T}^{2}} \partial_{t} \rho_{m} \cdot h-\int_{\mathbb{T}^{2}} G\left(D_{N}\left(\rho_{m}\right) D_{N}\left(\boldsymbol{w}_{m}\right)\right) \cdot \nabla h+k \int_{\mathbb{T}^{2}} \nabla \rho_{m} \cdot \nabla h=\int_{\mathbb{T}^{2}} g \cdot h,
\end{aligned}
$$

for all $(\boldsymbol{v}, h) \in L^{2}\left(0, T_{m} ; \mathbf{V}_{m}\right) \times L^{2}\left(0, T_{m} ; V^{m}\right)$. Actually $T_{m}=T$ (see [9]), and this concludes the construction of the approximate solutions $\left\{\left(\boldsymbol{w}_{m}, \rho_{m}\right)\right\}_{m \in \mathbb{N}}$.

In what follows, to keep the notation concise, se set $\boldsymbol{w}=\boldsymbol{w}_{m}$ and $\rho=\rho_{m}$. Also, we use the same notation $\|\cdot\|$ for both $\|\cdot\|_{H^{0}}$ and $\|\cdot\|_{H_{0}}$.

3.2. Estimates for $\left\|A^{1 / 2} D_{N}^{1 / 2} \rho(t)\right\|$ and $\left\|A^{1 / 2} D_{N}^{1 / 2} \boldsymbol{w}(t)\right\|$. We set $\Lambda:=(-\Delta)^{1 / 2}$ and note that $\|\Lambda \boldsymbol{u}\|=\|\nabla \boldsymbol{u}\|$. We test the Equation (7) against $A D_{N} \rho$ (this means that we take the scalar product in $\mathrm{L}^{2}(\Omega)$ ), with $\Omega=\mathbb{T}^{2}$, and obtain, using the Cauchy-Schwarz inequality,

$$
\begin{aligned}
\frac{1}{2} \frac{\mathrm{d}}{\mathrm{d} t}\left\|A^{1 / 2} D_{N}^{1 / 2} \rho\right\|^{2}+k\left\|\nabla A^{1 / 2} D_{N}^{1 / 2} \rho\right\|^{2} & \leq\left|\left(\Lambda A^{1 / 2} D_{N}^{1 / 2} \rho, \Lambda^{-1} A^{1 / 2} D_{N}^{1 / 2} g\right)\right| \\
& \leq \frac{k}{2}\left\|\nabla A^{1 / 2} D_{N}^{1 / 2} \rho\right\|^{2}+\frac{1}{2 k}\left\|\Lambda^{-1} A^{1 / 2} D_{N}^{1 / 2} g\right\|^{2} .
\end{aligned}
$$

Setting $y(t)=\left\|A^{1 / 2} D_{N}^{1 / 2} \rho(t)\right\|^{2}$ and using the Poincaré inequality, we thus obtain

$$
y^{\prime}(t)+k \lambda_{1} y(t) \leq \frac{1}{k}\left\|\Lambda^{-1} A^{1 / 2} D_{N}^{1 / 2} g\right\|^{2} .
$$

Applying the Gronwall lemma, we deduce

$$
y(t) \leq \frac{1}{k^{2} \lambda_{1}}\left\|\Lambda^{-1} A^{1 / 2} D_{N}^{1 / 2} g\right\|^{2}+\left(y(0)-\frac{1}{k^{2} \lambda_{1}}\left\|\Lambda^{-1} A^{1 / 2} D_{N}^{1 / 2} g\right\|^{2}\right) \mathrm{e}^{-k \lambda_{1} t}=: R_{1}(t)^{2},
$$

so that

$$
\limsup _{t \rightarrow+\infty} y(t) \leq \lim _{t \rightarrow+\infty} R_{1}(t)^{2}=\frac{1}{k^{2} \lambda_{1}}\left\|\Lambda^{-1} A^{1 / 2} D_{N}^{1 / 2} g\right\|^{2}=: \frac{r_{1}^{2}}{2}
$$

This means that $y(t)$ and $R_{1}(t)^{2}$ enter a ball of radius $r_{1}^{2}$ after long enough time. 
Then, we test the Equation (6) against $A D_{N} \boldsymbol{w}$, obtaining

$$
\begin{aligned}
\frac{1}{2} \frac{\mathrm{d}}{\mathrm{d} t}\left\|A^{1 / 2} D_{N}^{1 / 2} \boldsymbol{w}\right\|^{2}+\nu\left\|\nabla A^{1 / 2} D_{N}^{1 / 2} \boldsymbol{w}\right\|^{2} & \leq\left\|A^{1 / 2} D_{N}^{1 / 2} \boldsymbol{w}\right\| \cdot\left\|A^{1 / 2} D_{N}^{1 / 2} \rho\right\| \\
& \leq\left\|\nabla A^{1 / 2} D_{N}^{1 / 2} \boldsymbol{w}\right\| \cdot \lambda_{1}^{-1 / 2}\left\|A^{1 / 2} D_{N}^{1 / 2} \rho\right\| \\
& \leq \frac{\nu}{2}\left\|\nabla A^{1 / 2} D_{N}^{1 / 2} \boldsymbol{w}\right\|^{2}+\frac{1}{2 \nu \lambda_{1}}\left\|A^{1 / 2} D_{N}^{1 / 2} \rho\right\|^{2} .
\end{aligned}
$$

Setting $z(t)=\left\|A^{1 / 2} D_{N}^{1 / 2} \boldsymbol{w}(t)\right\|^{2}$ and using the Poincaré inequality, we get

$$
z^{\prime}(t)+\nu \lambda_{1} z(t) \leq \frac{1}{\nu \lambda_{1}}\left\|A^{1 / 2} D_{N}^{1 / 2} \rho(t)\right\|^{2} \leq \frac{r_{1}^{2}+2 y(0) \mathrm{e}^{-k \lambda_{1} t}}{2 \nu \lambda_{1}}
$$

If we multiply both members by $\mathrm{e}^{\nu \lambda_{1} t}$, integrate in time on $[0, t]$ and then divide by $\mathrm{e}^{\nu \lambda_{1} t}$, we deduce

$$
z(t) \leq z(0) \mathrm{e}^{-\nu \lambda_{1} t}+\frac{r_{1}^{2}}{2 \nu^{2} \lambda_{1}^{2}}\left(1-\mathrm{e}^{-\nu \lambda_{1} t}\right)+\frac{y(0)}{\nu \lambda_{1}} \mathrm{e}^{-\nu \lambda_{1} t} \int_{0}^{t} \mathrm{e}^{(\nu-k) \lambda_{1} s} \mathrm{~d} s .
$$

We need to distinguish two cases: $\nu=k$ and $\nu \neq k$. If $\nu=k$, we have

$$
z(t) \leq \frac{r_{1}^{2}}{2 \nu^{2} \lambda_{1}^{2}}+\left(z(0)-\frac{r_{1}^{2}}{2 \nu^{2} \lambda_{1}^{2}}+\frac{y(0)}{\nu \lambda_{1}} t\right) \mathrm{e}^{-\nu \lambda_{1} t} .
$$

If $\nu \neq k$, then

$$
z(t) \leq \frac{r_{1}^{2}}{2 \nu^{2} \lambda_{1}^{2}}+\left(z(0)-\frac{r_{1}^{2}}{2 \nu^{2} \lambda_{1}^{2}}\right) \mathrm{e}^{-\nu \lambda_{1} t}+\frac{y(0)}{\nu(\nu-k) \lambda_{1}^{2}}\left(\mathrm{e}^{-k \lambda_{1} t}-\mathrm{e}^{-\nu \lambda_{1} t}\right) .
$$

In any case, we obtain

$$
\begin{aligned}
& z(t) \leq R_{2}(t)^{2}:= \begin{cases}\frac{r_{1}^{2}}{2 \nu^{2} \lambda_{1}^{2}}+\left(z(0)-\frac{r_{1}^{2}}{2 \nu^{2} \lambda_{1}^{2}}+\frac{y(0)}{\nu \lambda_{1}} t\right) \mathrm{e}^{-\nu \lambda_{1} t} & \text { if } \nu=k, \\
\frac{r_{1}^{2}}{2 \nu^{2} \lambda_{1}^{2}}+\left(z(0)-\frac{r_{1}^{2}}{2 \nu^{2} \lambda_{1}^{2}}\right) \mathrm{e}^{-\nu \lambda_{1} t}+\frac{y(0)}{\nu(\nu-k) \lambda_{1}^{2}}\left(\mathrm{e}^{-k \lambda_{1} t}-\mathrm{e}^{-\nu \lambda_{1} t}\right) & \text { if } \nu \neq k,\end{cases} \\
& \limsup _{t \rightarrow+\infty} z(t) \leq \lim _{t \rightarrow+\infty} R_{2}(t)^{2}=\frac{r_{1}^{2}}{2 \nu^{2} \lambda_{1}^{2}}=: \frac{r_{2}^{2}}{2} .
\end{aligned}
$$

This means that $z(t)$ and $R_{2}(t)^{2}$ enter a ball of radius $r_{2}^{2}$ after long enough time. If we set $r:=$ $\max \left\{r_{1}, r_{2}\right\}$, we deduce that there exists $t_{r}>0$ such that

$$
\left\|A^{1 / 2} D_{N}^{1 / 2} \rho(t)\right\| \leq R_{1}(t) \leq r, \text { and }\left\|A^{1 / 2} D_{N}^{1 / 2} \boldsymbol{w}(t)\right\| \leq R_{2}(t) \leq r
$$

for every $t \geq t_{r}$.

3.3. Estimates for $\left\|A D_{N}^{1 / 2} \rho(t)\right\|$ and $\left\|A D_{N}^{1 / 2} \boldsymbol{w}(t)\right\|$. In what follows we look for higher order estimates. This time, we start by testing the Equation (6) by $A^{2} D_{N} \boldsymbol{w}$ and noticing that the nonlinear term cancels out (this holds true just in the $2 \mathrm{D}$ periodic case), so that

$$
\begin{gathered}
\frac{1}{2} \frac{\mathrm{d}}{\mathrm{d} t}\left\|A D_{N}^{1 / 2} \boldsymbol{w}\right\|^{2}+\nu\left\|\nabla A D_{N}^{1 / 2} \boldsymbol{w}\right\|^{2} \leq\left|\left(A^{1 / 2} D_{N}^{1 / 2} \rho, A^{3 / 2} D_{N}^{1 / 2} \boldsymbol{w}\right)\right| \\
\leq \frac{\nu}{2}\left\|A^{3 / 2} D_{N}^{1 / 2} \boldsymbol{w}\right\|^{2}+\frac{1}{2 \nu}\left\|A^{1 / 2} D_{N}^{1 / 2} \rho\right\|^{2},
\end{gathered}
$$


and hence

$$
\frac{\mathrm{d}}{\mathrm{d} t}\left\|A D_{N}^{1 / 2} \boldsymbol{w}\right\|^{2}+\nu\left\|\nabla A D_{N}^{1 / 2} \boldsymbol{w}\right\|^{2} \leq \frac{R_{1}^{2}}{\nu} .
$$

Setting $Z(t)=\left\|A D_{N}^{1 / 2} \boldsymbol{w}(t)\right\|^{2}$ and using the Poincaré inequality, we have $Z^{\prime}(t)+\nu \lambda_{1} Z(t) \leq$ $R_{1}(t)^{2} / \nu$. Multiplying by $\mathrm{e}^{\nu \lambda_{1} t}$ and integrating on $\left[t_{r}, t\right]$, with $t \geq t_{r}$, where $R_{1}(t) \leq r$, we obtain

$$
\begin{gathered}
Z(t) \leq Z\left(t_{r}\right) \mathrm{e}^{-\nu \lambda_{1}\left(t-t_{r}\right)}+\frac{r^{2}}{\nu^{2} \lambda_{1}}\left(1-\mathrm{e}^{-\nu \lambda_{1}\left(t-t_{r}\right)}\right) \\
\limsup _{t \rightarrow+\infty} Z(t) \leq \frac{r^{2}}{\nu^{2} \lambda_{1}}=: \frac{s_{2}^{2}}{2} .
\end{gathered}
$$

Now, we test the Equation (77) against $A^{2} D_{N} \rho$, obtaining (here the nonlinear term does not disappear):

$$
\frac{1}{2} \frac{\mathrm{d}}{\mathrm{d} t}\left\|A D_{N}^{1 / 2} \rho\right\|^{2}+k\left\|A^{3 / 2} D_{N}^{1 / 2} \rho\right\|^{2} \leq\left|\left(A^{1 / 2} D_{N}^{1 / 2} g, A^{3 / 2} D_{N}^{1 / 2} \rho\right)\right|+\left|\left(D_{N} \boldsymbol{w} \cdot \nabla D_{N} \rho, A D_{N} \rho\right)\right| .
$$

Then we observe that

$$
\left|\left(A^{1 / 2} D_{N}^{1 / 2} g, A^{3 / 2} D_{N}^{1 / 2} \rho\right)\right| \leq \frac{k}{4}\left\|A^{3 / 2} D_{N}^{1 / 2} \rho\right\|^{2}+\frac{1}{k}\left\|A^{1 / 2} D_{N}^{1 / 2} g\right\|^{2}
$$

and

$$
\begin{aligned}
& \left|\left(D_{N} \boldsymbol{w} \cdot \nabla D_{N} \rho, A D_{N} \rho\right)\right|=\left|\left(D_{N} \boldsymbol{w} \cdot \nabla A D_{N} \rho, D_{N} \rho\right)\right| \leq\left\|D_{N} \boldsymbol{w}\right\|_{\mathrm{L}^{4}}\left\|\nabla A D_{N} \rho\right\|\left\|D_{N} \rho\right\|_{\mathrm{L}^{4}} \\
& \quad \leq c_{4}\left\|D_{N} \boldsymbol{w}\right\|^{1 / 2}\left\|\nabla D_{N} \boldsymbol{w}\right\|^{1 / 2}\left\|D_{N} \rho\right\|^{1 / 2}\left\|\nabla D_{N} \rho\right\|^{1 / 2}\left\|\nabla A D_{N} \rho\right\| .
\end{aligned}
$$

Here, we are using the Ladyzhenskaja inequality $\|u\|_{L^{4}} \leq c_{4}\|u\|_{L^{2}}^{1 / 2}\|\nabla u\|_{L^{2}}^{1 / 2}$ for a scalar function $u$ defined on a torus $\mathbb{T}^{2}$. Note that, if $\boldsymbol{u}=\left(u_{1}, u_{2}\right)$ is a vector field, still defined on $\mathbb{T}^{2}$, and we set $\|\boldsymbol{u}\|_{\mathrm{L}^{p}}^{p}=\left\|u_{1}\right\|_{\mathrm{L}^{p}}^{p}+\left\|u_{2}\right\|_{\mathrm{L}^{p}}^{p}$, we have also $\|\boldsymbol{u}\|_{\mathrm{L}^{4}} \leq c_{4}\|\boldsymbol{u}\|_{\mathrm{L}^{2}}^{1 / 2}\|\nabla \boldsymbol{u}\|_{\mathrm{L}^{2}}^{1 / 2}$ (with the same constant $c_{4}>0$ ). Using

$$
\left\|D_{N} \boldsymbol{w}\right\| \leq\left\|A^{1 / 2} D_{N}^{1 / 2} \boldsymbol{w}\right\|, \text { and }\left\|\nabla D_{N} \boldsymbol{w}\right\| \leq \frac{(N+1)^{1 / 2}}{\alpha}\left\|A^{1 / 2} D_{N}^{1 / 2} \boldsymbol{w}\right\|
$$

and similar inequalities, we deduce

$$
\begin{gathered}
\left|\left(D_{N} \boldsymbol{w} \cdot \nabla D_{N} \rho, A D_{N} \rho\right)\right| \leq c_{4}^{2} \frac{N+1}{\alpha^{2}}\left\|A^{1 / 2} D_{N}^{1 / 2} \boldsymbol{w}\right\|\left\|A^{1 / 2} D_{N}^{1 / 2} \rho\right\|\left\|A^{3 / 2} D_{N}^{1 / 2} \rho\right\| \\
\leq \frac{k}{4}\left\|A^{3 / 2} D_{N}^{1 / 2} \rho\right\|^{2}+\frac{c_{4}^{4}(N+1)^{2}}{k \alpha^{4}}\left\|A^{1 / 2} D_{N}^{1 / 2} \boldsymbol{w}\right\|^{2}\left\|A^{1 / 2} D_{N}^{1 / 2} \rho\right\|^{2} .
\end{gathered}
$$

Substituting above and multiplying by 2 , we obtain

$$
\begin{aligned}
\frac{\mathrm{d}}{\mathrm{d} t}\left\|A D_{N}^{1 / 2} \rho\right\|^{2}+k\left\|A^{3 / 2} D_{N}^{1 / 2} \rho\right\|^{2} & \leq \frac{2}{k}\left\|A^{1 / 2} D_{N}^{1 / 2} g\right\|^{2}+\frac{2 c_{4}^{4}(N+1)^{2}}{k \alpha^{4}}\left\|A^{1 / 2} D_{N}^{1 / 2} \boldsymbol{w}\right\|^{2}\left\|A^{1 / 2} D_{N}^{1 / 2} \rho\right\|^{2} \\
& \leq \frac{2}{k}\left\|A^{1 / 2} D_{N}^{1 / 2} g\right\|^{2}+\frac{2 c_{4}^{4}(N+1)^{2}}{k \alpha^{4}} R_{1}^{2} R_{2}^{2} \\
& \leq \frac{2}{k}\left\|A^{1 / 2} D_{N}^{1 / 2} g\right\|^{2}+\frac{2 c_{4}^{4}(N+1)^{2}}{k \alpha^{4}} r^{4}=: \beta \quad \text { for } t \geq t_{r} .
\end{aligned}
$$


Setting $Y(t)=\left\|A D_{N}^{1 / 2} \rho\right\|^{2}$ and applying the Poincaré inequality, we have $Y^{\prime}(t)+k \lambda_{1} Y(t) \leq \beta$ (when $t \geq t_{r}$ ). Proceeding as before, we deduce

$$
\begin{aligned}
& Y(t) \leq Y\left(t_{r}\right) \mathrm{e}^{-k \lambda_{1}\left(t-t_{r}\right)}+\frac{\beta}{k \lambda_{1}}\left(1-\mathrm{e}^{-k \lambda_{1}\left(t-t_{r}\right)}\right), \\
& \limsup _{t \rightarrow+\infty} Y(t) \leq \frac{\beta}{k \lambda_{1}}=: \frac{s_{1}^{2}}{2} .
\end{aligned}
$$

If we set $s:=\max \left\{s_{1}, s_{2}\right\}$, we conclude that there exists $t_{s} \geq t_{r}>0$ such that

$$
\left\|A D_{N}^{1 / 2} \rho(t)\right\| \leq s, \text { and }\left\|A D_{N}^{1 / 2} \boldsymbol{w}(t)\right\| \leq s
$$

for every $t \geq t_{s}$.

\section{EXISTENCE OF AN INERTIAL MANIFOLD}

To perform our analysis, we find convenient to rewrite system (6) - (8) as

$$
\begin{aligned}
& \partial_{t} \overline{\boldsymbol{v}}-\nu \Delta \overline{\boldsymbol{v}}+\nabla \cdot \overline{D_{N} \overline{\boldsymbol{v}} \otimes D_{N} \overline{\boldsymbol{v}}}+\nabla \bar{\pi}=\bar{\vartheta} \boldsymbol{e}_{2}, \\
& \partial_{t} \bar{\vartheta}-k \Delta \bar{\vartheta}+\nabla \cdot \overline{D_{N} \bar{\vartheta} D_{N} \overline{\boldsymbol{v}}}=g, \\
& \nabla \cdot \overline{\boldsymbol{v}}=0 \\
& \boldsymbol{w}=A^{-1} \boldsymbol{v}=: \overline{\boldsymbol{v}} \\
& \rho=A^{-1} \vartheta=: \bar{\vartheta}
\end{aligned}
$$

and applying the operator $A$, term by term, to these equations we get the equivalent problem

$$
\begin{aligned}
& \partial_{t} \boldsymbol{v}-\nu \Delta \boldsymbol{v}+\nabla \cdot\left(D_{N} \overline{\boldsymbol{v}} \otimes D_{N} \overline{\boldsymbol{v}}\right)+\nabla \pi=\vartheta \boldsymbol{e}_{2}, \\
& \partial_{t} \vartheta-k \Delta \vartheta+\nabla \cdot\left(D_{N} \bar{\vartheta} D_{N} \overline{\boldsymbol{v}}\right)=f, \\
& \nabla \cdot \boldsymbol{v}=0,
\end{aligned}
$$

with initial conditions

$$
\boldsymbol{v}(0, x)=\boldsymbol{u}_{0}(x), \text { and } \vartheta(0, t)=\theta_{0}(x),
$$

and $f(\boldsymbol{x})=A g(\boldsymbol{x})$ as introduced in (2).

Set $B(\boldsymbol{v}, \boldsymbol{w}):=\nabla \cdot(\overline{\boldsymbol{v}} \otimes \overline{\boldsymbol{w}}), \mathcal{R}_{1}(\boldsymbol{v}, \vartheta):=B\left(D_{N} \overline{\boldsymbol{v}}, D_{N} \overline{\boldsymbol{v}}\right), \mathcal{R}_{2}(\boldsymbol{v}, \vartheta):=\nabla \cdot\left(D_{N} \bar{\vartheta} D_{N} \overline{\boldsymbol{v}}\right), \mathcal{R}:=$ $\left(\mathcal{R}_{1}, \mathcal{R}_{2}\right), \boldsymbol{V}:=(\boldsymbol{v}, \vartheta) \in\left(\mathrm{L}^{2}(\Omega)\right)^{3}$, and $\eta=(\nu, \nu, k)$. Then, equations (24)-(25) can be written as

$$
\partial_{t} \boldsymbol{V}-\eta \cdot \Delta \boldsymbol{V}+\mathcal{R}(\boldsymbol{V})=\left(\vartheta \boldsymbol{e}_{2}, f\right) .
$$

In order to prove the existence of the inertial manifold, we show that $\mathcal{R}:\left(\mathrm{L}^{2}(\Omega)\right)^{3} \rightarrow\left(\mathrm{L}^{2}(\Omega)\right)^{3}$ is locally Lipschitz continuous in $\left(\mathrm{L}^{2}(\Omega)\right)^{3}$. We have that

$$
\begin{aligned}
\left\|\mathcal{R}_{1}\left(\boldsymbol{V}_{1}\right)-\mathcal{R}_{1}\left(\boldsymbol{V}_{2}\right)\right\| & =\left\|B\left(D_{N} \overline{\boldsymbol{v}}_{1}, D_{N} \overline{\boldsymbol{v}}_{1}\right)-B\left(D_{N} \overline{\boldsymbol{v}}_{2}, D_{N} \overline{\boldsymbol{v}}_{2}\right)\right\| \\
& \leq\left\|B\left(D_{N} \overline{\boldsymbol{v}}_{1}, D_{N}\left(\overline{\boldsymbol{v}}_{1}-\overline{\boldsymbol{v}}_{2}\right)\right)\right\|+\left\|B\left(D_{N}\left(\overline{\boldsymbol{v}}_{1}-\overline{\boldsymbol{v}}_{2}\right), D_{N} \overline{\boldsymbol{v}}_{2}\right)\right\| \\
& \leq\left\|D_{N} \overline{\boldsymbol{v}}_{1}\right\|_{L^{4}}\left\|\nabla D_{N}\left(\overline{\boldsymbol{v}}_{1}-\overline{\boldsymbol{v}}_{2}\right)\right\|_{L^{4}}+\left\|D_{N}\left(\overline{\boldsymbol{v}}_{1}-\overline{\boldsymbol{v}}_{2}\right)\right\|_{L^{4}}\left\|\nabla D_{N} \overline{\boldsymbol{v}}_{2}\right\|_{L^{4}} \\
& \leq c \lambda_{1}^{-1}\left(\left\|A D_{N} \overline{\boldsymbol{v}}_{1}\right\|+\left\|A D_{N} \overline{\boldsymbol{v}}_{2}\right\|\right)\left\|A D_{N}\left(\overline{\boldsymbol{v}}_{1}-\overline{\boldsymbol{v}}_{2}\right)\right\| \\
& \leq c \lambda_{1}^{-1}\left(\left\|D_{N} \boldsymbol{v}_{1}\right\|+\left\|D_{N} \boldsymbol{v}_{2}\right\|\right)\left\|D_{N}\left(\boldsymbol{v}_{1}-\boldsymbol{v}_{2}\right)\right\| \\
& \leq c \lambda_{1}^{-1}(N+1)^{3 / 2}\left(\left\|D_{N}^{1 / 2} \boldsymbol{v}_{1}\right\|+\left\|D_{N}^{1 / 2} \boldsymbol{v}_{2}\right\|\right)\left\|\boldsymbol{V}_{1}-\boldsymbol{V}_{2}\right\|,
\end{aligned}
$$


and

$$
\begin{aligned}
\left\|\mathcal{R}_{2}\left(\boldsymbol{V}_{1}\right)-\mathcal{R}_{2}\left(\boldsymbol{V}_{2}\right)\right\|= & \left\|\nabla \cdot\left(D_{N} \bar{\vartheta}_{1} D_{N} \overline{\boldsymbol{v}}_{1}\right)-\nabla \cdot\left(D_{N} \bar{\vartheta}_{2} D_{N} \overline{\boldsymbol{v}}_{2}\right)\right\| \\
\leq & \left\|\nabla \cdot D_{N}\left(\bar{\vartheta}_{1}-\bar{\vartheta}_{2}\right) D_{N} \overline{\boldsymbol{v}}_{1}\right\|+\left\|\nabla \cdot D_{N}\left[\bar{\vartheta}_{2} D_{N}\left(\overline{\boldsymbol{v}}_{1}-\overline{\boldsymbol{v}}_{2}\right)\right]\right\| \\
\leq & c \lambda_{1}^{-1}\left(\left\|A D_{N} \overline{\boldsymbol{v}}_{1}\right\|\left\|A D_{N}\left(\bar{\vartheta}_{1}-\bar{\vartheta}_{2}\right)\right\|\right. \\
& \left.+\left\|D_{N} \vartheta_{2}\right\|\left\|D_{N}\left(\boldsymbol{v}_{1}-\boldsymbol{v}_{2}\right)\right\|\right) \\
\leq & c \lambda_{1}^{-1}\left(\left\|D_{N} \boldsymbol{v}_{1}\right\|\left\|D_{N}\left(\vartheta_{1}-\vartheta_{2}\right)\right\|+\left\|D_{N} \vartheta_{2}\right\|\left\|D_{N}\left(\boldsymbol{v}_{1}-\boldsymbol{v}_{2}\right)\right\|\right) \\
\leq & c \lambda_{1}^{-1}(N+1)^{3 / 2}\left(\left\|D_{N}^{1 / 2} \boldsymbol{v}_{1}\right\|\left\|\vartheta_{1}-\vartheta_{2}\right\|\right. \\
& \left.+\left\|D_{N}^{1 / 2} \vartheta_{2}\right\|\left\|\boldsymbol{v}_{1}-\boldsymbol{v}_{2}\right\|\right) \\
\leq & c \lambda_{1}^{-1}(N+1)^{3 / 2}\left(\left\|D_{N}^{1 / 2} \boldsymbol{v}_{1}\right\|+\left\|D_{N}^{1 / 2} \vartheta_{2}\right\|\right)\left\|\boldsymbol{V}_{1}-\boldsymbol{V}_{2}\right\| .
\end{aligned}
$$

Thus, we have that

$$
\left\|\mathcal{R}\left(\boldsymbol{V}_{1}\right)-\mathcal{R}\left(\boldsymbol{V}_{2}\right)\right\| \leq c \lambda_{1}^{-1}(N+1)^{3 / 2}\left(2\left\|D_{N}^{1 / 2} \boldsymbol{v}_{1}\right\|+\left\|D_{N}^{1 / 2} \boldsymbol{v}_{2}\right\|+\left\|D_{N}^{1 / 2} \vartheta_{2}\right\|\right)\left\|\boldsymbol{V}_{1}-\boldsymbol{V}_{2}\right\|,
$$

and we set $\mathfrak{L}:=c \lambda_{1}^{-1}(N+1)^{3 / 2}$.

In reproducing the argument used in [1] to prove point (i) in [1, Proposition 3] (see also [1. Proposition 9]), i.e., the "cone invariance property", which is stated in Proposition 4.1-(i) below, we introduce the following truncated nonlinearities:

$$
\begin{gathered}
\mathcal{F}_{1}(\boldsymbol{V}):=\chi_{\varrho}\left(\left\|D_{N}^{1 / 2} \boldsymbol{V}\right\|\right)\left[\mathcal{R}_{1}(\boldsymbol{V})-\vartheta e_{2}\right], \mathcal{F}_{2}(\boldsymbol{V}):=\chi_{\varrho}\left(\left\|D_{N}^{1 / 2} \boldsymbol{V}\right\|\right)\left[\mathcal{R}_{2}(\boldsymbol{V})-f\right], \\
\text { and } \mathcal{F}:=\left(\mathcal{F}_{1}, \mathcal{F}_{2}\right) .
\end{gathered}
$$

Here, $\chi$ is a smooth cut-off function outside the ball of radius $\rho=2$ in $\left(\mathrm{L}^{2}(\Omega)\right)^{3}$. Indeed, let $\chi: \mathbb{R}^{+} \rightarrow[0,1]$ with $\chi(r)=1$ for $0 \leq r \leq 1, \chi(r)=0$ for $r \geq 2$, and $\left|\chi^{\prime}(r)\right| \leq 2$ for $r \geq 0$ (which is null outside the ball of radius $\rho=2 \tilde{\varrho})$. Define $\chi_{\tilde{\varrho}}(r):=\chi(r / \tilde{\varrho})$ for $r \geq 0$.

Consider

$$
\begin{aligned}
& \partial_{t} \boldsymbol{v}-\nu \Delta \boldsymbol{v}+\chi_{\tilde{\varrho}}\left(\left\|D_{N}^{1 / 2} \boldsymbol{V}\right\|\right)\left[\mathcal{R}_{1}(\boldsymbol{V})-\vartheta \boldsymbol{e}_{2}\right]=0, \\
& \partial_{t} \vartheta-k \Delta \vartheta+\chi_{\tilde{\varrho}}\left(\left\|D_{N}^{1 / 2} \boldsymbol{V}\right\|\right)\left[\mathcal{R}_{2}(\boldsymbol{V})-f\right]=0,
\end{aligned}
$$

or equivalently

$$
\partial_{t} \boldsymbol{V}+\eta \cdot A \boldsymbol{V}+\mathcal{F}(\boldsymbol{V})=0
$$

and observe that $\|\boldsymbol{v}\| \cong\left\|A^{-1 / 2} D_{N}^{1 / 2} \boldsymbol{v}\right\| \cong\left\|A^{1 / 2} D_{N}^{1 / 2} \overline{\boldsymbol{v}}\right\| \cong\left\|A^{1 / 2} D_{N}^{1 / 2} \boldsymbol{w}\right\|$ and similarly we have that $\|\vartheta\| \cong\left\|A^{-1 / 2} D_{N}^{1 / 2} \vartheta\right\| \cong\left\|A^{1 / 2} D_{N}^{1 / 2} \bar{\vartheta}\right\| \cong\left\|A^{1 / 2} D_{N}^{1 / 2} \rho\right\|$.

Let us recall that in the Section 3 (see the formulas in (23) $)$, we have shown that $\left\|D_{N}^{1 / 2} \boldsymbol{V}(t)\right\| \leq$ $C\left\|A^{1 / 2} D_{N}^{1 / 2} \boldsymbol{V}(t)\right\| \leq r=:$ : for sufficiently large time $t \geq t_{\tilde{\varrho}}$, where $r=\tilde{\varrho}$ is a suitable fixed radius and $C=C\left(\alpha, \lambda_{1}\right)$. Now, since $\mathcal{R}:\left(\mathrm{L}^{2}(\Omega)\right)^{3} \rightarrow\left(\mathrm{L}^{2}(\Omega)\right)^{3}$ is locally Lipschitz, with calculations analogous to those in (28) -(30), it can be proved that the truncated nonlinearity $\mathcal{F}$, defined in (31), is globally Lipschitz continuous with Lipschitz constant given by $\mathcal{L}:=c \lambda_{1}^{-1}(N+1)^{3 / 2} \tilde{\varrho}$.

4.1. Spectral gap condition and inertial manifolds. We now give the elements to prove our main result, i.e. the existence of an inertial manifold to the system (24)-(27) (which is equivalent to (6) -(8)) in the sense of Theorem 4.3 below. 
Since the nonlinearity of (32)-(33) is globally Lipschitz, we will prove that this system has the "strong squeezing property", stated precisely in Theorem 4.3, provided that an appropriate "spectral gap condition" (see Proposition4.1-(i), below) is verified. Indeed, for $\gamma>0$ and $n \in \mathbb{N}$, we introduce the cone

$$
\begin{aligned}
\Gamma_{n, \gamma}:=\left\{\left(\begin{array}{l}
\boldsymbol{V}_{1} \\
\boldsymbol{V}_{2}
\end{array}\right)=\left(\begin{array}{c}
\left(\boldsymbol{v}_{1}, \vartheta_{1}\right) \\
\left(\boldsymbol{v}_{2}, \vartheta_{2}\right)
\end{array}\right) \in \mathcal{H} \times \mathcal{H}:\left\|D_{N}^{1 / 2} Q_{n}\left(\left(\boldsymbol{v}_{1}, \vartheta_{1}\right)-\left(\boldsymbol{v}_{2}, \vartheta_{2}\right)\right)\right\|\right. \\
\left.\leq \gamma\left\|D_{N}^{1 / 2} P_{n}\left(\left(\boldsymbol{v}_{1}, \vartheta_{1}\right)-\left(\boldsymbol{v}_{2}, \vartheta_{2}\right)\right)\right\|\right\} .
\end{aligned}
$$

Here, we denote by $P_{n}$ the orthogonal projection from $\mathcal{H}=H_{0} \times H^{0}$ onto $\operatorname{span}\left\{\left(\phi_{i}, \varphi_{j}\right)\right\}$, where $\left\{\phi_{i}\right\}_{i=1}^{\infty}$ and $\left\{\varphi_{j}\right\}_{j=1}^{\infty}$ are orthonormal bases of $H_{0}$ and $H^{0}$, respectively, and also define $Q_{n}:=I-P_{n}$.

Loosely speaking, the strong squeezing property asserts that if the dynamics of two trajectories starts inside the cone $\Gamma_{n, \gamma}$, then the trajectories stay inside the cone forever, and the higher Fourier modes of the difference are dominated by the lower modes (i.e., the cone invariance property); on the other hand, for as long as the two trajectories are outside the cone, then the higher Fourier modes of the difference decay exponentially fast, i.e., the decay property (see Theorem 4.3 below for further details).

Accordingly, in the case of the system (32)-(33), we have the following result.

Proposition 4.1. Let $\mathcal{L}=c \lambda_{1}^{-1}(N+1)^{3 / 2} \varrho$ be the Lipschitz constant associated with the truncated nonlinearity $\mathcal{F}$ in (31). Let $\boldsymbol{V}_{1}=\left(\boldsymbol{v}_{1}, \vartheta_{1}\right)$ and $\boldsymbol{V}_{2}=\left(\boldsymbol{v}_{2}, \vartheta_{2}\right)$ be two solutions of (32)-(33). Then this system satisfies the following properties.

(i) The cone invariance property: Assume that $n$ is large enough such that the "spectral gap condition" $\lambda_{n+1}-\lambda_{n}>\frac{\mathcal{L}(\gamma+1)^{2}}{\nu \gamma}$ holds true. If $\boldsymbol{v}_{1}\left(t_{0}\right) \in \Gamma_{n, \gamma}$ for some $t_{0} \geq 0$, then we have $\left(\begin{array}{l}\left(\boldsymbol{v}_{1}(t), \vartheta_{1}(t)\right) \\ \left(\boldsymbol{v}_{2}(t), \vartheta_{2}(t)\right)\end{array}\right) \in \Gamma_{n, \gamma}$ for all $t \geq 0$.

(ii) The decay property: Assume that $n$ is large enough such that $\lambda_{n+1}>\nu^{-1} \mathcal{L}\left(\frac{1}{\gamma}+1\right)$. If $\left(\begin{array}{l}\left(\boldsymbol{v}_{1}(t), \vartheta_{1}(t)\right) \\ \left(\boldsymbol{v}_{2}(t), \vartheta_{2}(t)\right)\end{array}\right) \in \Gamma_{n, \gamma}$ for $0 \leq t \leq T$, then it holds

$\left\|D_{N}^{1 / 2} Q_{n}\left(\left(\boldsymbol{v}_{1}(t), \vartheta_{1}(t)\right)-\left(\boldsymbol{v}_{2}(t), \vartheta_{2}(t)\right)\right)\right\| \leq\left\|D_{N}^{1 / 2} Q_{n}\left(\left(\boldsymbol{v}_{1}(0), \vartheta_{1}(0)\right)-\left(\boldsymbol{v}_{2}(0), \vartheta_{2}(0)\right)\right)\right\| e^{-\beta_{n} t}$ for $0 \leq t \leq T$,

where $\beta_{n}=\lambda_{n+1}|\eta|-\mathcal{L}\left(\frac{1}{\gamma}+1\right)$

The proof of this proposition will be provided later in Subsection 4.2 .

Now, for the case at hand, we give the precise notion of Inertial manifold.

Definition 4.2 (Inertial manifold). Consider the solution operator $S(t)$ associated with system (32) -(33). A subset $\mathcal{M} \subseteq \mathcal{H}$ is called an initial manifold for (32)-(33) if the following properties are satisfied:

(i) $\mathcal{M}$ is a finite-dimensional Lipschitz manifold;

(ii) $\mathcal{M}$ is invariant, i.e., $S(t) \mathcal{M} \subset \mathcal{M}$ for all $t \leq 0$;

(iii) $\mathcal{M}$ attracts exponentially all the solutions of (32)-(33); i.e.,

$$
\operatorname{dist}\left(S(t)\left(\boldsymbol{v}_{0}, \vartheta_{0}\right), \mathcal{M}\right) \rightarrow 0 \text { as } t \rightarrow \infty
$$

for every $\left(\boldsymbol{v}_{0}, \vartheta_{0}\right) \in \mathcal{H}$, and the rate of decay in (36) is exponential, uniformly for $\left(\boldsymbol{v}_{0}, \vartheta_{0}\right)$ in bounded sets in $\mathcal{H}$. 
Property (iii) implies that $\mathcal{M}$ contains the global attractor.

Next, we state a fundamental theorem concerning the fact that the strong squeezing property implies the existence of an inertial manifold for dissipative evolution equations. There are a number of proofs of such a result that can be found, for instance, in [13, 18, 20].

Theorem 4.3. Consider a nonlinear evolutionary equation of the type $v_{t}+A v+N(v)=0$, where $A$ is a linear, unbounded self-adjoint positive operator, acting in a Hilbert space $H$, such that $A^{-1}$ is compact and $N: H \rightarrow H$ is a nonlinear operator. Assume that the solution $v(t)$ enters a ball in $H$ with radius $\varrho$ for sufficiently large time $t$. For $\gamma>0$ and $n \in \mathbb{N}$, we define the cone

$$
\Gamma_{n, \gamma}:=\left\{\left(\begin{array}{l}
v_{1} \\
v_{2}
\end{array}\right) \in H \times H:\left|Q_{n}\left(v_{1}-v_{2}\right)\right|_{H} \leq \gamma\left|P_{n}\left(v_{1}-v_{2}\right)\right|_{H}\right\} .
$$

Let $\chi$ be a smooth cut-off function outside the ball of radius $2 \varrho$ in $H$ and let $\chi_{\varrho}$ be defined as in (32) - (33). Assume that there exists $n \in \mathbb{N}$ such that the "prepared" equation

$$
v_{t}+A v+\chi_{\varrho}(|v|) N(v)=0
$$

satisfies the "strong squeezing property", i.e., for any two solutions $v_{1}$ and $v_{2}$ of the "prepared" equation (38), it holds that

(I) if $\left(\begin{array}{l}v_{1}\left(t_{0}\right) \\ v_{2}\left(t_{0}\right)\end{array}\right) \in \Gamma_{n, \gamma}$ for some $t_{0} \geq 0$, then $\left(\begin{array}{l}v_{1}(t) \\ v_{2}(t)\end{array}\right) \in \Gamma_{n, \gamma}$ for all $t \geq t_{0}$;

(II) if $\left(\begin{array}{l}v_{1}(t) \\ v_{2}(t)\end{array}\right) \notin \Gamma_{n, \gamma}$ for $0 \leq t \leq T$, then there exists $a_{n}>0$ such that

$$
\left|Q_{n}\left(v_{1}(t)-v_{2}(t)\right)\right|_{H} \leq\left|Q_{n}\left(v_{1}(0)\right)-v_{2}(0)\right|_{H} e^{a_{n} t}, \quad \text { for } 0 \leq t \leq T .
$$

Then, the "prepared" equation (38) admits an n-dimensional inertial manifold in $H$. In addition, the following exponential tracking property holds: for any $v_{0} \in H$, there exists a time $\tau \geq 0$ and a solution $S(t) \varphi_{0}$ on the inertial manifold such that

$$
\left|S(t+\tau) v_{0}-S(t) \varphi_{0}\right|_{H} \leq C e^{-a_{n} t},
$$

where the constant $C$ depends on $\left|S(\tau) v_{0}\right|_{H}$ and $\left|\varphi_{0}\right|_{H}$.

As a consequence of Proposition 4.1 the strong squeezing property is verified provided that $n \in \mathbb{N}$ is large enough; then in view of Theorem 4.3. we have the following result

Theorem 4.4. System (32)-(33) admits a finite dimensional inertial manifold $\mathcal{M}$ in $\mathcal{H}$, i.e., the solution $S(t)\left(\boldsymbol{u}_{0}, \theta_{0}\right)$ of (32)-(33) approaches the invariant Lipschitz manifold $\mathcal{M}$ exponentially fast in time.

4.2. Proof of Proposition 4.1, Let $\boldsymbol{V}_{1}=\left(\boldsymbol{v}_{1}, \vartheta_{1}\right)$ and $\boldsymbol{V}_{2}=\left(\boldsymbol{v}_{2}, \vartheta_{2}\right)$ be two solutions of (32)(33). To show the cone invariance, i.e. the condition given in Proposition 4.1-(i) (see also [1, Proposition 3-(i)]), it is enough to show that $\left(\begin{array}{l}\boldsymbol{V}_{1}(t) \\ \boldsymbol{V}_{2}(t)\end{array}\right)=\left(\begin{array}{l}\left(\boldsymbol{v}_{1}(t), \vartheta_{1}(t)\right) \\ \left(\boldsymbol{v}_{2}(t), \vartheta_{2}(t)\right)\end{array}\right)$ cannot pass through the boundary of the cone if the dynamics starts inside the cone. More precisely, we will prove that

$$
\frac{d}{d t}\left(\left\|D_{N}^{1 / 2} Q_{n}\left(\boldsymbol{V}_{1}(t)-\boldsymbol{V}_{2}(t)\right)\right\|-\gamma\left\|D_{N}^{1 / 2} P_{n}\left(\boldsymbol{V}_{1}(t)-\boldsymbol{V}_{2}(t)\right)\right\|\right)<0
$$

whenever $\left(\begin{array}{l}\boldsymbol{V}_{1}(t) \\ \boldsymbol{V}_{2}(t)\end{array}\right) \in \partial \tilde{\Gamma}_{n, \gamma}$, where $\partial \tilde{\Gamma}_{n, \gamma}$ stands for the boundary of the cone $\tilde{\Gamma}_{n, \gamma}$. 
From (32)-(33), taking the difference equations of $\boldsymbol{V}_{1}$ and $\boldsymbol{V}_{2}$, we have that

$$
\begin{aligned}
& \partial_{t}\left(\boldsymbol{v}_{1}-\boldsymbol{v}_{2}\right)-\nu \Delta\left(\boldsymbol{v}_{1}-\boldsymbol{v}_{2}\right)+\mathcal{F}_{1}\left(\boldsymbol{V}_{1}\right)-\mathcal{F}_{1}\left(\boldsymbol{V}_{2}\right)=0, \\
& \partial_{t}\left(\vartheta_{1}-\vartheta_{2}\right)-k \Delta\left(\vartheta_{1}-\vartheta_{2}\right)+\mathcal{F}_{2}\left(\boldsymbol{V}_{1}\right)-\mathcal{F}_{2}\left(\boldsymbol{V}_{2}\right)=0,
\end{aligned}
$$

or more compactly

$$
\partial_{t}\left(\boldsymbol{V}_{1}-\boldsymbol{V}_{2}\right)+\eta \cdot A\left(\boldsymbol{V}_{1}-\boldsymbol{V}_{2}\right)+\mathcal{F}\left(\boldsymbol{V}_{1}\right)-\mathcal{F}\left(\boldsymbol{V}_{2}\right)=0 .
$$

By setting $\boldsymbol{p}:=P_{n}\left(\boldsymbol{V}_{1}-\boldsymbol{V}_{2}\right)$, and $\boldsymbol{q}:=Q_{n}\left(\boldsymbol{V}_{1}-\boldsymbol{V}_{2}\right)$, we obtain

$$
\begin{aligned}
& \boldsymbol{p}_{t}+\eta \cdot A \boldsymbol{p}+P_{n}\left(\mathcal{F}\left(\boldsymbol{V}_{1}\right)-\mathcal{F}\left(\boldsymbol{V}_{2}\right)\right)=0, \\
& \boldsymbol{q}_{t}+\eta \cdot A \boldsymbol{q}+Q_{n}\left(\mathcal{F}\left(\boldsymbol{V}_{1}\right)-\mathcal{F}\left(\boldsymbol{V}_{2}\right)\right)=0 .
\end{aligned}
$$

Take the $L^{2}$-product of (41) with $D_{N} \boldsymbol{p}$, to get

$$
\frac{1}{2} \frac{d}{d t}\left\|D_{N}^{1 / 2} \boldsymbol{p}\right\|^{2}+|\eta|\left\|A^{1 / 2} D_{N}^{1 / 2} \boldsymbol{p}\right\|^{2}+\left(D_{N}^{1 / 2} P_{N}\left(\mathcal{F}\left(\boldsymbol{V}_{1}\right)-\mathcal{F}\left(\boldsymbol{V}_{2}\right)\right), D_{N}^{1 / 2} \boldsymbol{p}\right)=0
$$

Thus by (30), which is now a global property due to the presence of $\chi_{\varrho}$, we have (with $\lambda_{n}$ large enough), we find

$$
\begin{aligned}
\frac{1}{2} \frac{d}{d t}\left\|D_{N}^{1 / 2} \boldsymbol{p}\right\|^{2} & \geq-\lambda_{n}|\eta|\left\|D_{N}^{1 / 2} \boldsymbol{p}\right\|^{2}-c\left\|D_{N}^{1 / 2}\left(\mathcal{F}\left(\boldsymbol{V}_{1}\right)-\mathcal{F}\left(\boldsymbol{V}_{2}\right)\right)\right\|\left\|D_{N}^{1 / 2} \boldsymbol{p}\right\| \\
& \geq-\lambda_{n}|\eta|\left\|D_{N}^{1 / 2} \boldsymbol{p}\right\|^{2}-\tilde{c}\left\|D_{N}^{1 / 2}\left(\boldsymbol{V}_{1}-\boldsymbol{V}_{2}\right)\right\|\left\|D_{N}^{1 / 2} \boldsymbol{p}\right\|
\end{aligned}
$$

with $\tilde{c}=c \lambda_{1}^{-1}(N+1)^{3 / 2} \tilde{\varrho}=\mathcal{L}$.

Without loss of generality, we can assume $\left\|D_{N}^{1 / 2} \boldsymbol{p}(t)\right\|>0$. Otherwise, if $\left\|D_{N}^{1 / 2} \boldsymbol{p}\left(t^{*}\right)\right\|=0$ for some $t^{*}$, then since we consider the boundary of the cone, we can assume $\left\|D_{N}^{1 / 2} \boldsymbol{q}\left(t^{*}\right)\right\|=$ $\gamma\left\|D_{N}^{1 / 2} \boldsymbol{p}\left(t^{*}\right)\right\|=0$, and thus $D_{N}^{1 / 2} \boldsymbol{V}_{1}\left(t^{*}\right)=D_{N}^{1 / 2} \boldsymbol{V}_{2}\left(t^{*}\right)$. By the uniqueness of solutions, we obtain $D_{N}^{1 / 2} \boldsymbol{V}_{1}(t)=D_{N}^{1 / 2} \boldsymbol{V}_{2}(t)$, for all $t \geq t^{*}$, and the cone invariance property follows. Thus $\left\|D_{N}^{1 / 2} \boldsymbol{p}(t)\right\|>0$, and we can divide both sides of (44) by $\left\|D_{N}^{1 / 2} \boldsymbol{p}(t)\right\|$, to reach

$$
\frac{d}{d t}\left\|D_{N}^{1 / 2} \boldsymbol{p}\right\| \geq-\lambda_{n}|\eta|\left\|D_{N}^{1 / 2} \boldsymbol{p}\right\|-\mathcal{L}\left\|D_{N}^{1 / 2}\left(\boldsymbol{V}_{1}-\boldsymbol{V}_{2}\right)\right\|
$$

Similarly, taking the $L^{2}$-inner product of (42) against $D_{N} \boldsymbol{q}$, we infer

$$
\frac{d}{d t}\left\|D_{N}^{1 / 2} \boldsymbol{q}\right\| \leq-\lambda_{n+1}|\eta|\left\|D_{N}^{1 / 2} \boldsymbol{q}\right\|+\mathcal{L}\left\|D_{N}^{1 / 2}\left(\boldsymbol{V}_{1}-\boldsymbol{V}_{2}\right)\right\|
$$

Now, multiplying equation (45) by $\gamma$ and subtracting it from (46), we obtain, exploiting the fact that $\boldsymbol{p}+\boldsymbol{q}=\boldsymbol{V}_{1}-\boldsymbol{V}_{2}$, the following relation

$$
\begin{aligned}
\frac{d}{d t}\left(\left\|D_{N}^{1 / 2} \boldsymbol{q}\right\|-\gamma\left\|D_{N}^{1 / 2} \boldsymbol{p}\right\|\right) \leq|\eta|\left(\lambda_{n} \gamma\left\|D_{N}^{1 / 2} \boldsymbol{p}\right\|-\lambda_{n+1}\left\|D_{N}^{1 / 2} \boldsymbol{q}\right\|\right) \\
+\mathcal{L}(\gamma+1)\left(\left\|D_{N}^{1 / 2} \boldsymbol{p}\right\|+\left\|D_{N}^{1 / 2} \boldsymbol{q}\right\|\right) .
\end{aligned}
$$

Thus, whenever $\left\|D_{N}^{1 / 2} \boldsymbol{q}(t)\right\|=\gamma\left\|D_{N}^{1 / 2} \boldsymbol{p}(t)\right\|$, that is $\left(\begin{array}{l}\boldsymbol{V}_{1}(t) \\ \boldsymbol{V}_{2}(t)\end{array}\right) \in \partial \tilde{\Gamma}_{n, \gamma}$, we have that

$$
\frac{d}{d t}\left(\left\|D_{N}^{1 / 2} \boldsymbol{q}\right\|-\gamma\left\|D_{N}^{1 / 2} \boldsymbol{p}\right\|\right) \leq\left(|\eta|\left(\lambda_{n}-\lambda_{n+1}\right)+\mathcal{L} \frac{(\gamma+1)^{2}}{\gamma}\right)\left\|D_{N}^{1 / 2} \boldsymbol{q}\right\|<0
$$


due to the assumption $\lambda_{n+1}-\lambda_{n}>\mathcal{L}(\gamma+1)^{2} /|\eta| \gamma$. Whence

$$
\frac{d}{d t}\left(\left\|D_{N}^{1 / 2} \boldsymbol{q}\right\|-\gamma\left\|D_{N}^{1 / 2} \boldsymbol{p}\right\|\right)<0
$$

this concludes point (i).

Now, to prove the decay property (ii), assume that $\left(\begin{array}{l}\boldsymbol{V}_{1}(t) \\ \boldsymbol{V}_{2}(t)\end{array}\right) \notin \tilde{\Gamma}_{n, \gamma}$ for $0 \leq t \leq T$, then $\left\|D_{N}^{1 / 2} \boldsymbol{q}(t)\right\|>\gamma\left\|D_{N}^{1 / 2} \boldsymbol{p}(t)\right\|$ for $0 \leq t \leq T$, and from (46) it follows that

$$
\begin{aligned}
\frac{d}{d t}\left\|D_{N}^{1 / 2} \boldsymbol{q}\right\| & \leq-\lambda_{n+1}|\eta|\left\|D_{N}^{1 / 2} \boldsymbol{q}\right\|+\tilde{c}(\|\boldsymbol{p}\|+\|\boldsymbol{q}\|) \\
& \leq-\lambda_{n+1}|\eta|\left\|D_{N}^{1 / 2} \boldsymbol{q}\right\|+\hat{c}\left(\left\|D_{N}^{1 / 2} \boldsymbol{p}\right\|+\left\|D_{N}^{1 / 2} \boldsymbol{q}\right\|\right) \\
& \leq-\left[\lambda_{n+1}|\eta|-\mathcal{L}\left(\frac{1}{\gamma}+1\right)\right]\left\|D_{N}^{1 / 2} q\right\|=-\beta_{n}\left\|D_{N}^{1 / 2} q\right\|,
\end{aligned}
$$

for $0 \leq t \leq T$, where $\beta_{n}=\lambda_{n+1}|\eta|-\mathcal{L}\left(\frac{1}{\gamma}+1\right)$. By Gronwall's inequality, we have that

$$
\left\|D_{N}^{1 / 2} \boldsymbol{q}(t)\right\| \leq\left\|D_{N}^{1 / 2} \boldsymbol{q}(0)\right\| e^{-\beta_{n} t}, \text { for } 0 \leq t \leq T .
$$

This concludes the proof.

Acknowledgments. The authors are members of the Gruppo Nazionale per l'Analisi Matematica, la Probabilità e le loro Applicazioni (GNAMPA) of the Istituto Nazionale di Alta Matematica (INdAM).

\section{REFERENCES}

[1] Abu Hamed, M., Guo, Y., Titi, E. S. Inertial manifolds for certain subgrid-scale $\alpha$-models of turbulence., SIAM J. Appl. Dyn. Syst. 14 (2015), no. 3, 1308-1325.

[2] N. A. Adams, S. Stolz, Deconvolution methods for subgrid-scale approximation in large eddy simulation, in: Modern Simulation Strategies for Turbulent Flow, R.T. Edwards, 2001.

[3] L. C. Berselli, L. Bisconti On the structural stability of the Euler-Voigt and Navier-Stokes-Voigt models, Nonlinear Anal. 75 (2012), no. 1, 117-130.

[4] L. C. Berselli, D. Catania, On the well-posedness of the Boussinesq equations with anisotropic filter for turbulent flows, Z. Anal. Anwend. 34 (2015), no. 1, 61-83.

[5] L. C. Berselli, D. Catania, On the Boussinesq equations with anisotropic filter in a vertical pipe, Dyn. Partial Differ. Equ. 12 (2015), no. 2, 177-192.

[6] L. C. Berselli, D. Catania R. Lewandowski, Convergence of approximate deconvolution models to the mean magnetohydrodynamics equations: Analysis of two models J. Math. Anal. Appl. 401 (2013) 864-880.

[7] L. C. Berselli, T. Iliescu, W. J. Layton, Mathematics of Large Eddy Simulation of Turbulent Flows, Scientific Computation, Springer-Verlag, Berlin, 2006.

[8] L. C. Berselli, R. Lewandowski, Convergence of approximate deconvolution models to the mean Navier-Stokes equations, Ann. Inst. H. Poincaré Anal. Non Linéaire 29 (2012) 171-198.

[9] L. Bisconti, On the convergence of an approximate deconvolution model to the $3 D$ mean Boussinesq equations, Math. Methods Appl. Sci., 38 (2015), no. 7, 1437-1450.

[10] L. Bisconti, D. Catania, Remarks on global attractors for the $3 D$ Navier-Stokes equations with horizontal filtering, Discrete Contin. Dyn. Syst. Ser. B 20 (2015), no. 1, 59-75.

[11] L. Bisconti, D. Catania, Global well-posedness of the two-dimensional horizontally filtered simplified Bardina turbulence model on a strip-like region, Commun. Pure Appl. Anal. 16, no 5, (2017) pp. 1861-1881

[12] V. V. Chepyzhov, M. I. Vishik, Nonautonomous evolution equations and their attractors. Russ. J. Math. Phys. 1 (1993), 165-190.

[13] P. Constantin, C. Foias, B. Nicolaenko, and R. Temam, Integral manifolds and inertial manifolds for dissipative partial differential equations, Applied Mathematical Sciences 70, Springer-Verlag, New York, 1989. 
[14] C. R. Doering, J. D. Gibbon, Applied analysis of the Navier-Stokes equations, Cambridge Texts in Applied Mathematics, Cambridge University Press, Cambridge, 1995.

[15] B. Guo, Nonlinear Galerkin methods for solving two dimensional Newton-Boussinesq equations, Chin. Ann. Math., Ser. B 16 (1995), no. 3, 379-390.

[16] H. Bessaih F. Flandoli, Weak attractor for a dissipative Euler equation, J. Dynamics Diff. Equations, 12 (2000), $713-732$.

[17] C. Foias, G. R. Sell, and R. Temam, Variétés inertielles des équations différentielles dissipatives [Inertial manifolds for dissipative differential equations], C. R. Acad. Sci. Paris Sér. I Math., 301 (1985), pp. 139-141.

[18] C. Foias, B. Nicolaenko, G. R. Sell, and R. Temam, Inertial manifolds for the Kuramoto-Sivashinsky equation and an estimate of their lowest dimension, J. Math. Pures Appl. (9) 67 (1988), 197-226.

[19] C. Foias, G. R. Sell, and R. Temam, Inertial manifold for nonlinear evolutionary equations, J. Differential Equations, 73 (1988), pp. 309-353.

[20] C. Foias, G. R. Sell, and E. S. Titi, Exponential Tracking and Approximation of Inertial Manifolds for Dissipative Nonlinear Equations, Journal of Dynamics and Differential Equation 1 (1989), pp. 199-244.

[21] R. Lewandowski, On a continuous deconvolution equation for turbulence models, Lecture Notes of Neças Center for Mathematical Modeling 5 (2009) 62-102.

[22] J.-L. Lions, Quelques méthodes de résolution des problèmes aux limites non linéaire, Dunod, Gauthier-Villars, Paris, 1969.

[23] S. Stolz, N. A. Adams, An approximate deconvolution procedure for large-eddy simulation, Phys. Fluids 11 (7) (1999) 1699-1701.

[24] S. Stolz, N. A. Adams, L. Kleiser, An approximate deconvolution model for large-eddy simulation with application to incompressible wall-bounded flows, Phys. Fluids 13 (4) (2001) 997-1015.

[25] R. Temam, Infinite Dimensional Dynamical Systems in Mechanics and Physics, Applied Mathematical Sciences, Vol. 68, Springer Verlag, New York, (1988).

[26] S. Zelik, Inertial manifolds and finite-dimensional reduction for dissipative PDEs, Proc. Roy. Soc. Edinburgh Sect. A 144 (2014), no. 6, 1245-1327. 